\title{
Plasma Characteristic Metabolites of Pediatric Community Acquired Pneumonia in TCM Syndrome Differentiation Syndrome Differentiation
}

\section{Li An}

Jiangsu Key Laboratory of Pediatric Respiratory Disease; Institute of Pediatrics

Lili Lin

Jiangsu Province Key Laboratory of Pediatric Respiratory Disease;Institute of Pediatrics

Shouchuan Wang

Affiliated Hospital of Nanjing University of Traditional Chinese Medicine

\section{Xia Zhao}

Jiangsu Key Laboratory of Atmospheric Environment Monitoring and Pollution Control

\section{Tong Xie}

Jiangsu Key Laboratory of Pediatric Respiratory Disease; Institute of Pediatrics

\section{Yan Yang}

Beijing Children's Hospital Affiliated to Capital Medical University

\section{Wensheng Zhai}

the First Affiliated Hospital of Henan University of TCM

\section{Lina Du}

Beijing Children's Hospital Affiliated to Capital Medical University

\section{Weiwei Li}

Jiangsu Key Laboratory of Pediatric Respiratory Disease; Institute of Pediatric

\section{Cunsi Shen}

Jiangsu Key Laboratory of Pediatric Respiratory Disease; Institute of Pediatrics

jianjian Ji

Jiangsu Key Laboratory of Pediatric Respiratory Disease; Institute of Pediatrics

\section{Ying Zhang}

UC Davis Genome Center: University of California Davis Genome Center

Jinjun Shan ( $\square$ jshan@njucm.edu.cn)

Jiangsu Key Laboratory of Pediatric Respiratory Disease, Institute of Pediatrics, Affiliated Hospital of Nanjing Universit y of Chinese Medicine, Nanjing 210023, China https://orcid.org/0000-0001-8079-7950

\section{Research}

Keywords: Community-acquired pneumonia, metabolomics, TCM, heat or cold syndrome type 
Posted Date: January 11th, 2021

DOl: https://doi.org/10.21203/rs.3.rs-141954/v1

License: (c) (i) This work is licensed under a Creative Commons Attribution 4.0 International License. Read Full License 


\section{Abstract}

Background Cold syndrome CS and Heat syndrome ( H S)S), with the opposite clinical manifestations, are two main syndrome types of pediatric community acquired pneumonia (in Traditional Chinese medicine TCM )). According to research of syndrome types among CAP children, about $2.92 \%$ of them can be identified with CS while $91.25 \%$ with HS. This study aim s to analyze plasma metabolic profiles and find out potential biomarkers for distinguishing $\mathrm{H} S$ from CS.

Methods A total of 296 patients and 55 healthy controls $(H C)$ were divided into discovery group $(n=213$, $H S=160, C S=23, H C=30$ ) and validation group $N=138, H S=93, C S=20, H C=$ Plasma metabolic profiles were detected by ultra performance liquid chromatography combined with linear ion trap quadrupoleOrbitrap mass spectrometry (UPLC/LTQ Orbitrap MS) in both positive and negative mode. Finally, plasma metabolic profiles were obtained through principal component analysis (PCA) and orthogonal partial least squares discriminant analysis (OPLS DA)DA). Differential metabolites in CS and HS were screened by counting the value of fold change (FC 1.5 or $0.667 \mathrm{P}$ value ( $\mathrm{P} 0.05$ ) and false discovery rate (FDR 0.05 Diagnostic accuracy of potential biomarkers was evaluated by receiver operating characteristic (ROC) curve.

Results Among all metabolites 3 amino acids including alanine, phenylalanine, arginine, and 4 glycerophospholipids including lysoPC20:1, lysoPE16:0, lysoPE18:0, PE(16:0 22:6) were increased (FC 1.5 FDR 0.05) in CS versus HC. While 6 glycerophospholipids including PC(18:1 18:1), PC(20:4 20:4), lysoPE 20:4, lysoPE 18:2, lysoPE 22:6 and

PE(16:0 18:2) were decreased (FC 0. 667 FDR 0.05) in HS versus HC. However, Ceramide(d18:1 24:1) was increased in HS versus HC. Differential metabolites were mainly involved in amino acid, glycerophospholipid and linoleic acid metabolism via KEGG pathway analysis $\mathrm{P}<0.05$ ) and they all exhibited good diagnostic abilities with ROC analysis AUC 0.954 Mo reover, lyso PC20:1, lysoPE16:0, lysoPE18:2, lysoPE20:4, lysoPE22:6, PC(18:1_18:1), PC(20:4_20: and PC(P 16:0_22:4) may serve as potential biomarkers for distinguishing HS from CS.

Conclusions Plasma metabolism of amino acids and lipids (triglyceride, glycerophospholipid and sphingomyelin were largely disturbed in CAP children with CS or HS. Among them, detection of glycerophospholipids via metabolomics can help diagnose these two syndrome types on clinic.

\section{Full Text}

This preprint is available for download as a PDF.

\section{Figures}




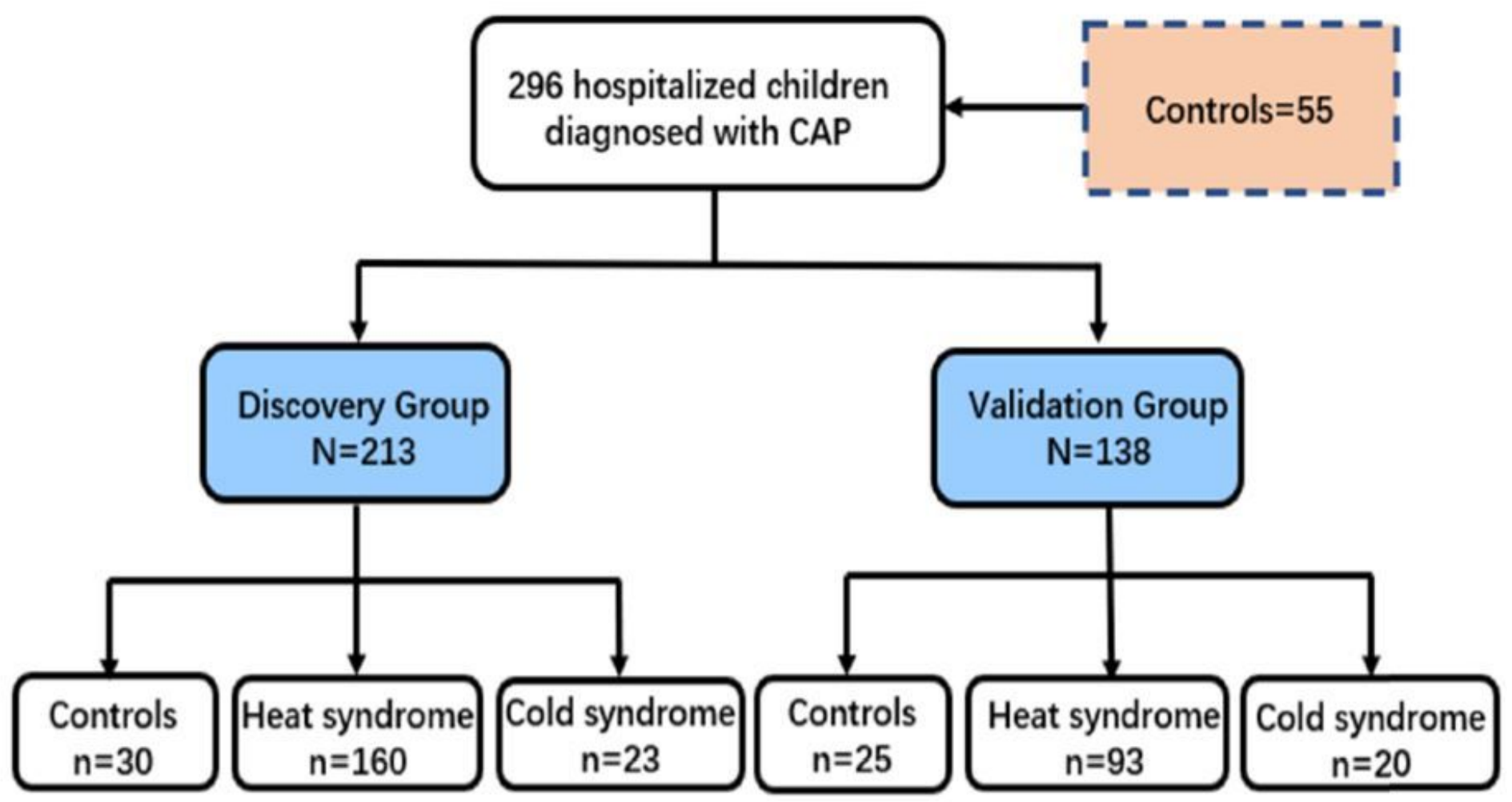

Figure 1

Flow chart of children enrolled in this study. Abbreviations: CAP=community acquired pneumonia. 

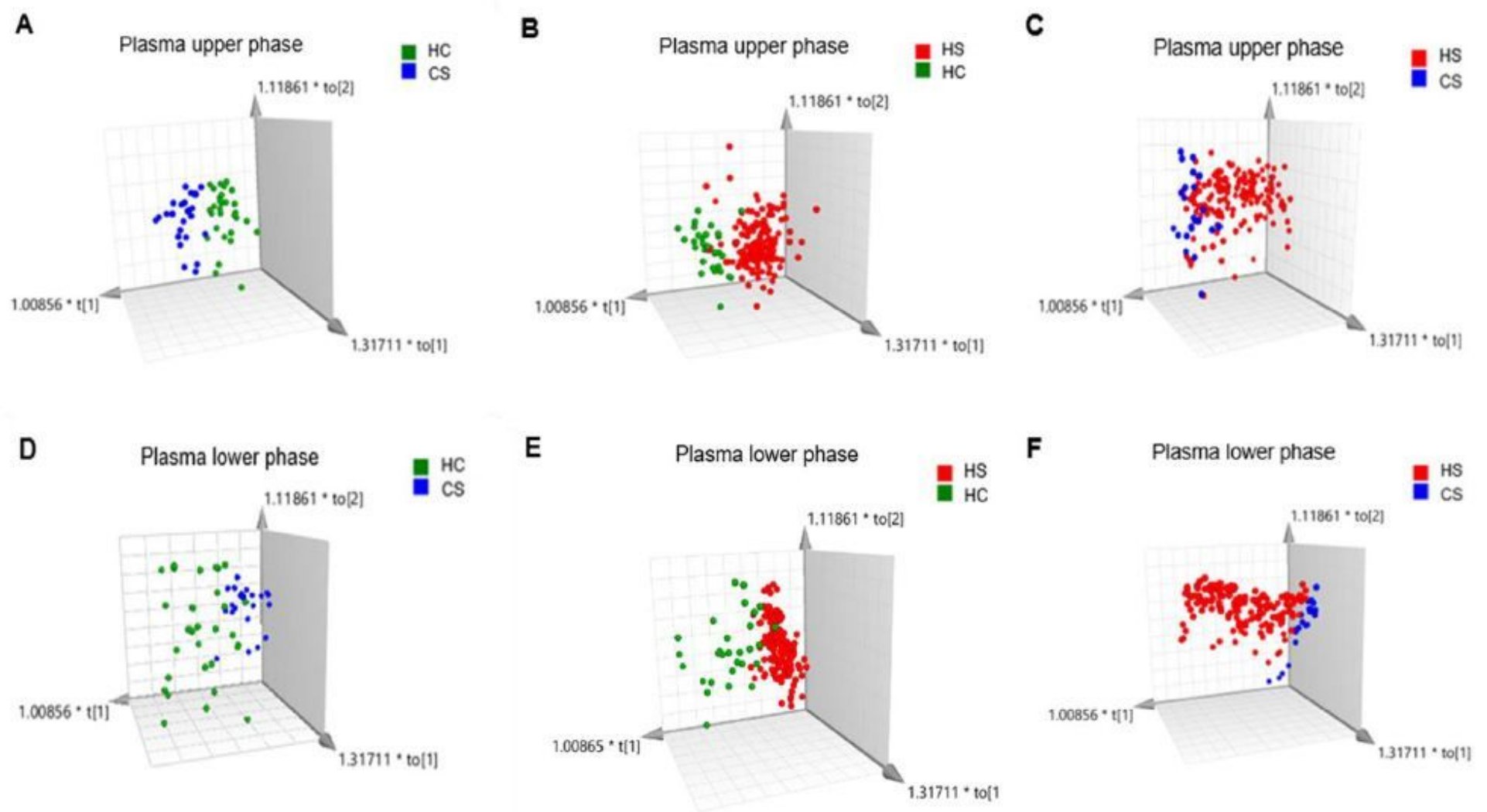

\section{Figure 2}

Metabolic profiles of plasma samples between CS, HS versus HC. A OPLS DA sc ore plots of plasma upper phase between CS versus HC. B OPLS DA score plots of plasma upper phase between HS versus HC. C OPLS DA score plots of plasma upper phase between CS versus HS. D OPLS DA score plots of plasma lower phase between CS versus HC. E OP LS DA score plots of plasma lower phase between HS versus HC. F OPLS DA score plots of plasma lower phase between CS versus HS. Elliptical area represents $95 \% \mathrm{Cl}$ of Hotelling's T squared distribution in each figure. R2Y indicates goodness of fit, Q2 indicates goodness of prediction. Abbreviations: $\mathrm{CS}=$ cold syndrome, $\mathrm{HS}=$ heat syndrome, $\mathrm{HC}=$ healthy control , OPLS DA=orthogonal partial least squares discriminant analysis, C I=confidence interval. 


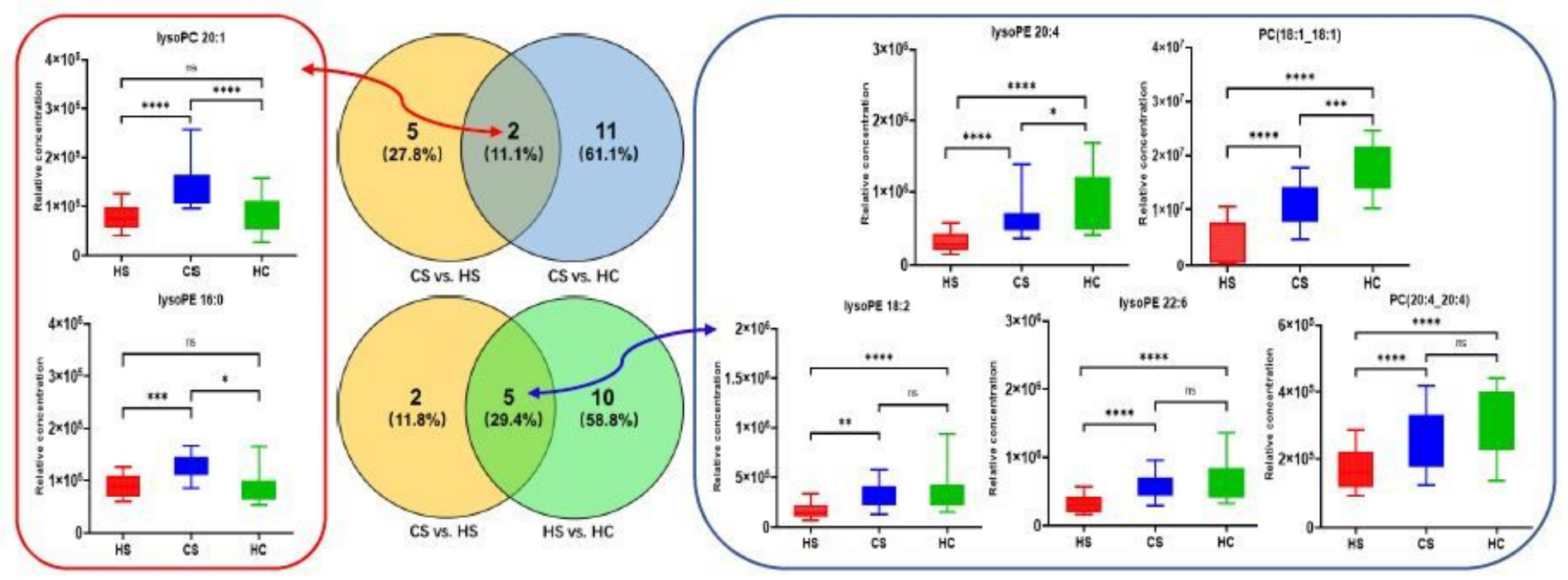

Figure 3

Analysis of differential metabolites screened in CS, HS versus HC. Venn diagram of differential metabolites in CS vs. HC, HS vs. HC and CS vs. HS groups. Each cycle in Venn diagram represent s 7, 13, 15 differential metabolites screened in CS vs. HS, CS vs. HC, HS vs. HC. The overlapping area represents the same differential metabolites in different groups. Box whisker $p$ lots showed the relative concentration of 7 differential metabolites for distinguishing CS $\mathrm{f}$ rom HS. $\mathrm{X}$ axis means grouping and $\mathrm{Y}$ axis means relative concentration. Whiskers are 1090 percentile range and boxes represent interquartile range $. P<0.05, P<0.01$, $* \star \star P<0.001, P<0.0001$ Abbreviations: ns=not significant, HS=heat syndrome, $\mathrm{CS}=$ cold syndrome, $\mathrm{HC}=$ healthy control, $\mathrm{PC}=$ phosphatidylcholine, $\mathrm{PE}=$ phosphatidylethanol amine, lysoPC=lysophosphatidylcholine, lysoPE=lysophosphatidylethanolamine.
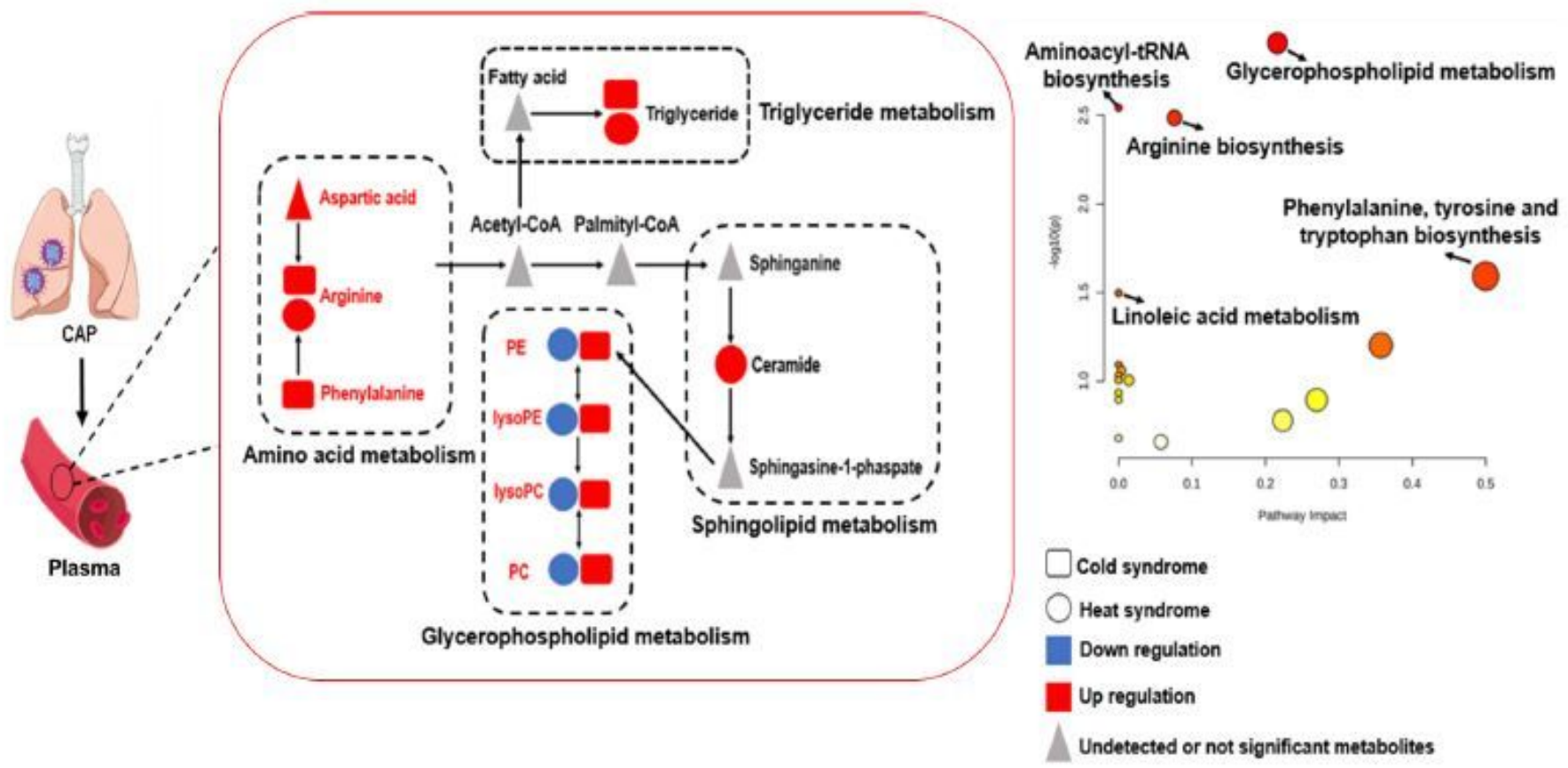


\section{Figure 4}

KEGG pathway analysis and metabolic pathway network of differential metabolites screened in $\mathrm{HS}$ and CS. KEGG pathway analysis were summarized based on 24 screened metabolites (Pख0.05). Names of 5 enriched pathways were annotated beside each point (Pख0.05). Metabolic pathway network was drawn by reference to screened differential metabolites and KEGG pathway analysis. Rectangle represents $C S$, circle represents HS, blue color represents down-regulation, red color represents up-regulation, grey triangle represents undetected or not significant metabolites. Name of differential metabolites screened in $\mathrm{CS}$ and $\mathrm{HS}$ were all labeled in red. Abbreviations: $\mathrm{CAP}=$ community acquired pneumonia, $\mathrm{HC}=$ healthy control, CS=cold syndrome, $\mathrm{HS}=$ heat syndrome, $\mathrm{PC}=$ phosphatidylcholine, $\mathrm{PE}=$ phosphatidylethanolamine, lysoPC=lysophosphatidylcholine, lysoPE=lysophosphatidylethanolamine.
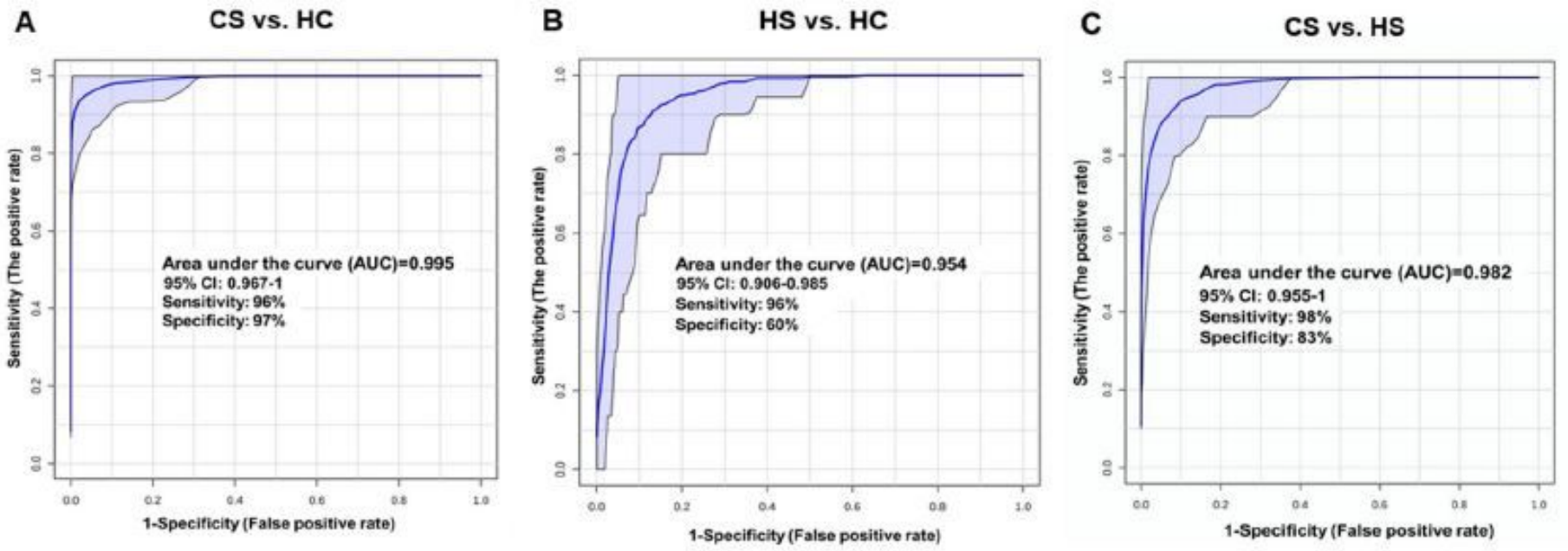

\section{Figure 5}

ROC curve for testing diagnostic significance of differential metabolites screened in CS and HS. A ROC curve of 13 differential metabolites screened i n CS vs. HC. 95\% Cl: 0.967 1, sensitivity: $96 \%$, specificity: 97\%, AUC: 0.995. B ROC curve of 15 differential metabolites screened in HS vs. HC. $95 \%$ Cl: 0.9060 .985 , sensitivity: $96 \%$, specificity: $60 \%$, AUC: 0.954 . C ROC curve of 7 differential metabolites screened in CS vs. HS. $95 \%$ Cl: 0.955 1, sensitivity: 98\%, specificity: 83\%, AUC: 0.982. Abbreviations: $\mathrm{HC}=$ healthy control, $\mathrm{CS}=$ cold syndrome, $\mathrm{HS}=$ heat syndrome, ROC receiver operating characteristic , AUC area under the curve, $\mathrm{Cl}=$ confidence interval.

\section{Supplementary Files}

This is a list of supplementary files associated with this preprint. Click to download.

- Graphicalabstract.jpg

- Supportinglnformation.pdf 